\title{
Period1 mediates rhythmic metabolism of toxins by interacting with CYP2E1
}

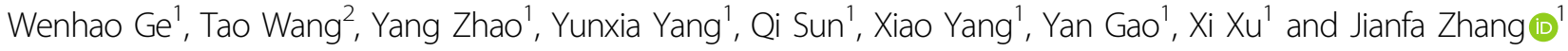

\begin{abstract}
The biological clock is an endogenous biological timing system, which controls metabolic functions in almost all organs. Nutrient metabolism, substrate processing, and detoxification are circadian controlled in livers. However, how the clock genes respond to toxins and influence toxicity keeps unclear. We identified the clock gene Per 1 was specifically elevated in mice exposed to toxins such as carbon tetrachloride $\left(\mathrm{CCl}_{4}\right)$. Mice lacking Per1 slowed down the metabolic rate of toxins including $\mathrm{CCl}_{4}$, capsaicin, and acetaminophen, exhibiting relatively more residues in the plasma. Liver injury and fibrosis induced by acute and chronic $\mathrm{CCl}_{4}$ exposure were markedly alleviated in Per1-deficient mice. These processes involved the binding of PER1 protein and hepatocyte nuclear factor-1alpha (HNF-1a), which enhances the recruitment of HNF-1a to cytochrome P450 2E1 (Cyp2e1) promoter and increases Cyp2e1 expression, thereby promoting metabolism for toxins in the livers. These results indicate that PER1 mediates the metabolism of toxins and appropriate suppression of Per1 response is a potential therapeutic target for toxin-induced hepatotoxicity.
\end{abstract}

\section{Introduction}

Most physiological processes in mammals display circadian rhythms that are driven by the endogenous circadian clock. Both central and peripheral clocks are operated by positive- and negative-feedback loops of circadian genes, such as Clock, Bmal1, Period1 (Per1), Period2 (Per2), Cryptochromes1 (Cry1) and Cryptochromes2 (Cry2), Rev-Erb- $\alpha$, and Rora. In the negative-feedback loops of circadian genes, the PER and CRY form complexes and shut down transcription initiated by CLOCK-BMAL1 heterodimers ${ }^{1,2}$. Circadian rhythms play an important role in maintaining homeostasis and solid organ function. Although disrupted rhythms can lead to metabolic impairments, the reverse is also true that disrupting metabolism can alter circadian rhythms ${ }^{3}$. Clock dysfunction accelerates the development of liver diseases such as fatty liver diseases, cirrhosis, hepatitis, and liver

\footnotetext{
Correspondence: Jianfa Zhang (jfzhang@mail.njust.edu.cn)

${ }^{1}$ Center for Molecular Metabolism, Nanjing University of Science \& Technology, Nanjing, China

${ }^{2}$ Cambridge Suda Genome Resource Center, Soochow University, Suzhou,

China

These authors contributed equally: Wenhao Ge, Tao Wang

Edited by M. Agostini
}

cancer, and these disorders also disrupt clock function ${ }^{4}$. On the other hand, patients with cirrhosis have liver portal hypertension and dysfunctional circadian clock systems ${ }^{5}$. Hepatic fibrosis induced by carbon tetrachloride $\left(\mathrm{CCl}_{4}\right)$ in mice leads to alterations in the circadian rhythms of hepatic clock genes ${ }^{6}$. The overarching evidence that circadian homeostasis is critical to human health, and conversely, that some abnormal metabolism and diseases induce abnormal responses of the circadian clock and negatively affect health, cannot be ignored.

Acute liver injury induced by hepatotoxins has been recognized as one of the most important pharmacovigilance concerns and the leading cause of drug withdrawal on safety grounds ${ }^{7,8}$. After acute liver injury, parenchymal cells regenerate and replace the necrotic or apoptotic cells. The wound-healing response of the liver to repeated injury leads to liver fibrosis. Advanced liver fibrosis results in cirrhosis, liver failure, and portal hypertension and often requires liver transplantation ${ }^{9}$. The generation of reactive oxygen species (ROS) and oxidative stress resulted from the metabolism of hepatotoxins is a common mechanism of liver injury. Of the multiple forms of P450s present in the liver endoplasmic 
reticulum, cytochrome P450 2E1 (CYP2E1) has been implicated as a key metabolizing enzyme for various xenobiotics from food and drugs into toxic metabolites which lead to liver injury. Induction of Cyp $2 e 1$ with ethanol, pyrazole, or other chemicals has been shown to promote oxidative stress ${ }^{10,11}$.

Although endogenous circadian controlled daily metabolism of toxins and hepatic Cyp2e1 expression have been described in mammals ${ }^{12,13}$, it still was unclear how the clock genes respond toxins, and how these responses influence the capacity to metabolize toxins. In present studies, we identified the clock gene Per1 was specifically elevated in mice exposed to toxins such as $\mathrm{CCl}_{4}$. We demonstrated the PER1 regulated Cyp2e1 expression, thereby promoting metabolism for toxins in the livers. These results suggest that PER1 is a regulator of liver metabolism of toxins. Appropriate suppression of the Per1 response is a potential therapeutic target for toxininduced hepatotoxicity and dysfunction of the circadian clock.

\section{Materials and methods}

\section{Animals and treatment}

Male, 8-10-week-old Per1 ${ }^{-1-}$ and WT C57BL/6 mice were used in this study. The Per $1^{-1-}$ mice used in this study have been characterized previously ${ }^{14}$. All mice were maintained under standard laboratory conditions, with 12-h light/12-h dark cycles and free access to food and water at all stages of the experiments. All procedures were approved by the Animal Care and Use Committee at Nanjing University of Science and Technology (ACUCNUST-20160016).

A single intraperitoneal injection of $\mathrm{CCl}_{4}(0.6 \mathrm{~mL} / \mathrm{kg}$ body weight) was administrated to WT and $P e r 1^{-1-}$ mice. As controls, animals received the same volume of olive oil intraperitoneally. To determine the statistical significance of any observed differences, we used five male mice per time point following $\mathrm{CCl}_{4}$ administration, which included 24,48 , and $72 \mathrm{~h}$. To detect the circadian mRNA expression of clock genes in response to $\mathrm{CCl}_{4}$, mice were killed at zeitgeber time (ZT)1 (ZT0 corresponds lights on and ZT12 to light off), ZT5, ZT9, ZT13, ZT17, ZT21, ZT24 (n $=5$, each time point). To determine the plasma pharmacokinetics of $\mathrm{CCl}_{4}$, the blood samples were obtained from five male mice per time point following $\mathrm{CCl}_{4}$ administration, which included 2, 4, 8, and $12 \mathrm{~h}$. For induction of liver fibrosis, $\mathrm{CCl}_{4}$ was injected twice weekly for 4 weeks. Mice were killed $48 \mathrm{~h}$ after the last injection. Serum aspartate transaminase (AST) and alanine transaminase (ALT) activity were measured using an AU2700 automatic biochemical analyzer (Olympus, Tokyo, Japan).

To determine the plasma pharmacokinetics of capsaicin, capsaicin was each separately suspended in $0.5 \%(\mathrm{w} / \mathrm{v})$ $\mathrm{CMC}-\mathrm{Na}$ solution to obtain a final concentration of
$30 \mathrm{mg} / \mathrm{ml}$. WT and Per1 ${ }^{-1-}$ mice were given capsaicin at a dose of $300 \mathrm{mg} / \mathrm{kg}$, via gavage. After oral administration, the blood samples $(0.6 \mathrm{~mL})$ were collected at the desired times (30 min, 1, and $3 \mathrm{~h}$ ) into heparinized centrifuge tubes.

To determine the plasma pharmacokinetics of acetaminophen (APAP), APAP was dissolved in warm saline and injected intraperitoneally at the dose of $500 \mathrm{mg} / \mathrm{kg}$ body weight, whereas saline was administered to control animals. After $0.5,1,2$, or $4 \mathrm{~h}$. Blood samples from six different mice per time point were then quickly centrifuged at $4{ }^{\circ} \mathrm{C}$ and a small plasma volume from each mouse was rapidly stored to assess the concentrations of APAP.

\section{Quantitation of $\mathrm{CCl}_{4}$ by the headspace gas chromatographic method}

Samples were prepared according to the method described by Jerry et al. ${ }^{15}$. Stoppered test tubes containing samples for $\mathrm{CCl}_{4}$ analysis were incubated at $60^{\circ} \mathrm{C}$ for $15 \mathrm{~min}$. All experiments were carried out by using Bruker GC-450 equipped with an electron capture detector (Bruker, Columbia, MD, USA). Column was $30 \mathrm{~m} \times$ $0.25 \mathrm{~mm}$ i.d. capillary coated with $0.50 \mathrm{~mm}$ of $50 \%$ phenyl-50\% methyl polysiloxane (Rxi-50). The chromatographic conditions were: column temperature, $100^{\circ} \mathrm{C}$ : detector temperature, $200^{\circ} \mathrm{C}$. Nitrogen was used as carrier gas.

\section{Determination of hepatic oxidative stress and GSH levels}

ROS was measured using 2', 7'-dichlorofluorescein diacetate as a probe. Lipid peroxidation was determined by measuring the formation of the thiobarbituric acidreactive substances spectrophotometrically and was expressed as malondialdehyde (MDA) concentration. Hepatic glutathione (GSH) levels were estimated by a colorimetric method using Ellman's reagent and glutathione reductase. Hepatic ROS, MDA, and GSH contents were assayed with commercial kits according to the manufacturers' instructions (Jiancheng, Nanjing, China).

\section{TUNEL staining}

At sacrifice, tissues were rapidly isolated, fixed in $4 \%$ paraformaldehyde, cryoprotected with $30 \%$ sucrose, and embedded in the optimal cutting temperature compound. The specimens were snap-frozen and sectioned into 15$\mu \mathrm{m}$ sagittal sections (CM1950; Leica, Germany). Terminal deoxynucleotidyl transferase dUTP nick end labeling (TUNEL) staining was performed using a TUNEL Apoptosis Assay Kit (Beyotime, C1088). Nuclei were stained with 4',6-diamidino-2-phenylindole (DAPI). Fluorescence images of liver slices were observed with fluorescence microscopy (Eclipse 800; Nikon, Tokyo, Japan). 


\section{Flow cytometry}

After treatment, cells were stained with annexin $\mathrm{V}$ and propidium iodide (PI) (Annexin V-early apoptosis detection kit, Beyotime Biotechnology) following the manufacturer's instructions. In brief, after culture under various conditions, cells were harvested and suspended in the appropriate binding buffer, stained with fluorescein isothiocyanate-conjugated annexin $\mathrm{V}$ and propidium iodide at room temperature for 15 minutes, and subsequently analyzed by a FACSCalibur flow cytometer (BD Biosciences, San Diego, CA, USA). Cells positive for annexin $\mathrm{V}$ and annexin $\mathrm{V} /$ propidium iodide were considered early and late apoptotic cells, respectively. All cells negative for annexin $\mathrm{V}$ were considered viable cells.

\section{Histological analysis}

Liver tissue was fixed in 10\% phosphate-buffered formalin overnight, embedded in paraffin, and cut into $4 \mu \mathrm{m}$ sections. Sections were stained with hematoxylin and eosin for routine examination or Masson's trichrome for visualization of hepatic collagen deposition. Immunohistochemical staining was performed according to standard procedures ${ }^{16}$ using an appropriate specific primary antibody (Anti-CYP2E1, Abcam, Cambridge, UK; Anti- $\alpha$ SMA, Millipore, Billerica, MA).

\section{Cells and treatment}

HepG2 E47 cells expressing Cyp2e1 (Boster Biological Technology Ltd., Wuhan, China) were cultured at $37^{\circ} \mathrm{C}$ under $5 \% \mathrm{CO}_{2}$ humidified atmosphere, using DMEM, supplemented with $10 \%$ FCS, $100 \mathrm{U} / \mathrm{ml}$ penicillin, and $100 \mathrm{mg} / \mathrm{ml}$ streptomycin.

Primary hepatocytes were isolated from 6 to 8-week-old male C57BL/6 mice using an in situ liver perfusion approach $^{17}$. In brief, hepatocytes were dissociated from anesthetized adult mice by non-recirculating collagenase perfusion (C5138, Sigma, USA) through the portal vein. The isolated cells are then filtered through a $100 \mu \mathrm{m}$ pore size mesh nylon filter. Cells were plated in collagen I-coated 6- or 12-well plated (at two or one million cells per well, respectively) in M199 medium plus 10\% fetal bovine serum plus penicillin/streptomycin. After 3 hours of attachment, the medium was replaced with the appropriate assay medium.

For Per1 knockdown experiments, the complementary oligonucleotide of small hairpin RNA targeting the $5^{\prime}$ GGTGCTCCCTAACTATCTATT- $3^{\prime}$ sequence was chemically synthesized, subcloned into the lentiviral vector (GenePharma, Shanghai, China), and transfected into primary hepatocytes or HepG2 E47 cells. Cells were infected with lentivirus at low multiplicity according to the manufacturer's instructions. Cells were selected in puromycin $(1 \mu \mathrm{g} / \mathrm{mL})$ and polyclonal populations were expanded and analyzed. A hairpin siRNA with no sequence homology to human genes provided by the manufacturer (GenePharma) was used as the negative control. Per1 mRNA knockdown was assessed by realtime PCR. Clones that had sufficient knockdown (>75\%) were used for further experiments.

For overexpression of human Per1, cells were transfected with pCMV-Sport2 Per1 or pCMV-Sport2 vector as control using Lipofectamine 2000 Transfection Reagent (Invitrogen, Carlsbad, CA). Construction of pCMV-Sport2 Per1 was characterized previously by Zheng and colleagues ${ }^{18}$.

The transfected cells were treated with $0.5 \%(\mathrm{v} / \mathrm{v}) \mathrm{CCl}_{4}$ in $0.25 \%$ dimethyl sulfoxide prepared in serum-free culture medium for $24 \mathrm{~h}$. All cell lines have been authenticated at the beginning of the study and again within 4 months after completion of the experiments. All cell lines have been tested for mycoplasma contamination frequently. Determination of MDA and GSH levels in cells were performed using commercial kits according to the manufacturers' instructions (Jiancheng, Nanjing, China).

\section{RNA extraction and quantitative real-time PCR}

Total RNA was extracted from samples with Karrol reagent (Karroten Scientific, Nanjing, China) according to the manufacturer's instructions. Reverse transcript reaction was carried out by reverse transcript kit (Invitrogen, Carlsbad, CA) according to the manufacturer's protocol. Real-time PCR was performed with the SYBR Green PCR Kit (Applied Biosystems, Foster City, CA) following the manufacturer's instructions on an ABI 7300 real-time PCR system (Applied Biosystems) in a 20- $\mu$ l volume. For an internal standard control, the expression level of glyceraldehyde-3-phosphate dehydrogenase (Gapdh) was simultaneously quantified. All primer sequences used for real-time PCR are shown in Table S1.

\section{RNA-sequencing for detection of differentially expressed genes and pathways}

RNA-sequencing (RNA-Seq) analysis and quantification were utilized to investigate changes in liver mRNA profiles among the different treatments performed. Isolated RNA was sent to BGI Co., Ltd. for conducting RNA-seq, which was performed on a BGISEQ-500 (Shenzhen, China). All samples were replicated three times for confirmation purposes. All the generated raw sequencing reads were filtered to remove reads with adapters, reads in which unknown bases are $>5 \%$, and low quality reads. Clean reads were then obtained and stored as FASTQ format. HISAT ${ }^{19}$ was used to map clean reads to the genome of GRCm38.p6. NOISeq ${ }^{20}$ method was used to screen deferentially expressed genes (DEGs). All DEGs were mapped to terms in Kyoto Encyclopedia of Genes and Genomes (KEGG) pathway enrichment analysis. 


\section{Western blot analysis}

Proteins were extracted following the procedure described previously ${ }^{21}$. The extraction was separated by sodium dodecyl sulfate polyacrylamide gel electrophoresis (SDS-PAGE) $8-12 \%$ polyacrylamide gel and then electrically transferred to a polyvinylidene difluoride membrane. After blocking with 5\% (w/v) BSA in TBST at room temperature for $1 \mathrm{~h}$, the membranes were then incubated with an appropriate specific primary antibody (AntiCYP2E1, Abcam, ab28146; anti-PER1, Abcam, ab3443; anti-HNF-1 $\alpha$, Cell Signaling Technology, \#89670; antiCBP, Cell Signaling Technology, \#7389; anti- $\beta$-ACTIN, Bioworld, AP0060) at $4{ }^{\circ} \mathrm{C}$ overnight, followed by incubation with HRP-conjugated secondary antibody (Boster Biological Technology Ltd., BA1054) and detected by enhanced chemical luminescence kit (Thermo scientific, Hudson, NH, USA).

\section{Immunoprecipitation assays}

Immunoprecipitation assays were performed as described previously ${ }^{22,23}$ with slight modification. For ChIP assays, cross-linked chromatin was immunoprecipitated with $5 \mu \mathrm{g}$ of antibody (anti-HNF-1 $\alpha$, Cell Signaling Technology, \#89670; anti-PER1, Abcam, ab3443), or negative control rabbit IgG (Beyotime, A7016) at $4{ }^{\circ} \mathrm{C}$ overnight. Immunoprecipitated DNA was then used as a template for PCR. All primer sequences used for ChIPPCRs were listed in Table S1.

For co-immunoprecipitations, liver tissues were homogenized and lysed with a Non-denaturing lysis buffer containing $20 \mathrm{mM}$ Tris- $\mathrm{HCl} \mathrm{pH}$ 8.0, $137 \mathrm{mM} \mathrm{NaCl}, 2 \mathrm{mM}$ ethylenediaminetetraacetic acid (EDTA), and 1\% NP-40 with protease inhibitor cocktail (Boster Biological Technology). To prepare immunoprecipitates, we incubated lysates with antibody (anti-HNF-1 $\alpha$, Cell Signaling Technology, \#89670; anti-PER1, Abcam, ab3443) overnight at $4{ }^{\circ} \mathrm{C}$, and then incubated with Protein ASepharose 4B (Invitrogen). Immunoprecipitates were washed five times with wash buffer containing $10 \mathrm{mM}$ Tris- $\mathrm{HCl} \mathrm{pH} \mathrm{7.4,} 150 \mathrm{mM} \mathrm{NaCl}, 1 \mathrm{mM}$ EGTA, $1 \mathrm{mM}$ EDTA, $1 \%$ Triton X-100, $0.2 \mathrm{mM}$ sodium orthovanadate with protease inhibitor cocktail, boiled in SDS-PAGE loading buffer. Proteins were analyzed by western blotting as described above.

\section{Quantitation of capsaicin by HPLC}

Samples were prepared according to the method described by Yingying Zhao et al. ${ }^{24}$. A $200 \mu \mathrm{L}$ aliquot of plasma was mixed with $50 \mu \mathrm{L} \alpha$-naphthol $(10 \mu \mathrm{g} / \mathrm{mL}$; Aladdin, China), $10 \mu \mathrm{L}$ methanol, $400 \mu \mathrm{L}$ water, and $500 \mu \mathrm{L}$ acetonitrile, and vortexing for $1 \mathrm{~min}$. The solution was then extracted with $1.5 \mathrm{~mL}$ each of acetic ether and cyclohexane for capsaicin, followed by vortexing for $3 \mathrm{~min}$. After $10 \mathrm{~min}$ centrifugation at $3000 \mathrm{rpm}, 2.5 \mathrm{ml}$ of the supernatant was transferred to another container and evaporated to dryness via nitrogen at $37^{\circ} \mathrm{C}$. The residue was dissolved in $300 \mu \mathrm{L}$ of acetonitrile and mixed for $1 \mathrm{~min}$. Following centrifugation at $3000 \mathrm{rpm}$ for $5 \mathrm{~min}$, $50 \mu \mathrm{L}$ of the supernatant was injected into the Highperformance liquid chromatography (HPLC) system for analysis.

Samples were analyzed using an HPLC (Waters 1525 System; Millipore, Bedford, MA, USA) on a reversedphase C18 column. The mobile phase was acetonitrile-water $(43: 57)$ at a flow rate of $1.0 \mathrm{~mL} / \mathrm{min}$. The elution of metabolites was monitored at a wavelength of $254 \mathrm{~nm}$.

\section{Quantitation of APAP by HPLC}

Samples were prepared according to a method described previously ${ }^{25}$. A $100 \mu \mathrm{g} / \mathrm{ml}$ methanol solution of theophylline was added to the plasma at a ratio of 1:1. This mixture was allowed to precipitate for at least $10 \mathrm{~min}$ and centrifuged twice at $11,000 \times g 6-7 \mathrm{~min}$ to pellet the precipitated proteins. The mixtures were filtered through $0.45-\mu \mathrm{m}$ HV Millipore cellulose filters and $20-\mu 1$ samples were injected immediately into the chromatographic system.

Samples were analyzed using an HPLC (Waters 1525 System; Millipore, Bedford, MA, USA) on a reversedphase $\mathrm{C} 18$ column. The gradient elution started with $30 \%$ methanol passing through the column at a flow-rate of $1.5 \mathrm{ml} / \mathrm{min}$. After a delay of $0.5 \mathrm{~min}$ the methanol concentration was increased linearly to $75 \%$ over $7.5 \mathrm{~min}$. The column was returned to $30 \%$ methanol after a delay of $1 \mathrm{~min}$. The variable-wavelength detector was set at $254 \mathrm{~nm}$.

\section{Statistics}

The single cosinor method was used for analysis of circadian rhythm ${ }^{26}$, and the cosine function equation was as follows: $Y(\mathrm{t})=\mathrm{M}+\mathrm{A} \cos (\mathrm{xt}+\mathrm{u})$. The rhythm characteristics estimated by this method included the mesor (middle value of the fitted cosine representing a rhythmadjusted mean), the amplitude (half the difference between the minimum and maximum of the fitted cosine function), and the acrophase (time of peak value in the fitted cosine function). Data were presented as means $\pm \mathrm{S}$. E.M. Statistical analysis was performed by Student's $t$ test, one-way ANOVA or two-way ANOVA followed by Tukey's post hoc test. Significance was defined as $P$ value $<0.05$. Sample sizes of all experiments were predetermined by calculations derived from our experience. No sample was excluded from the analyses. Animals were not randomly assigned during collection, but the strain, sex, and age of the mice were the same, and the data analysis was single masked. Investigators were not blinded to the group allocation during the experiment and 
outcome assessment. The number of replicates was indicated in each figure legend. All qPCR data represented the mean of three technical replicates. The mean of the technical replicates was used per biological replicate. All statistical tests justified as appropriate and the data met the assumptions of the tests. There was an estimate of variation within each group of data.

\section{Results}

RNA sequence analysis of gene expression in the liver of mice exposed to $\mathrm{CCl}_{4}$

To search possible response genes following toxins exposure, we performed RNA-Seq to analyze the hepatic gene expression changes between toxin $\mathrm{CCl}_{4}$-treated and oil-controlled mice. A KEGG pathway analysis showed that organismal systems pathways (KEGG level 1) were the major pathways altered in $\mathrm{CCl}_{4}$-treated liver and 2048 DEGs are involved in multiple pathways essential for $\mathrm{CCl}_{4}$-induced liver injury and fibrosis (Fig. 1A), and 70 DEGs in the environmental adaptation pathway on level 2 of the KEGG functional category (Fig. 1C). Circadian rhythm is one of the top 20 affected pathways in the environmental adaptation implicated by DEGs (Fig. 1B). The larger the Rich factor, the greater the enrichment. Circadian rhythm pathway showed the greatest enrichment (Fig. 1B). In KEGG level 3, 294 KEGG pathways including the circadian rhythm pathway between the two groups changed significantly (Supplement Data). A gene that draws our attention was that encoding for the mouse Per1, the core clock gene in negative-feedback loops (Fig. 1C, D). Circadian rhythm pathway contained 10 changed genes (Fig. 1D), eight genes were upregulated and two gene was downregulated (Fig. 1D). However, other core clock genes including Clock, Bmal1, Cry1, Cry2, and Per2, were no obvious changes, and only Per1 was specifically elevated in livers following $\mathrm{CCl}_{4}$ exposure.

Response changes of clock genes in mice exposed to $\mathrm{CCl}_{4}$

To validate the results obtained from RNA-Seq, we carried out real-time PCR analysis for core clock genes expression in the liver mRNA between $\mathrm{CCl}_{4}$ and control group. As shown in Fig. 2, in both experimental and control groups, the core clock gene expressions were highly significantly changed within 24 hours. The present results confirmed that $\mathrm{CCl}_{4}$ acutely increased the expression of Per1 in livers (Fig. 2C). Table 1 showed the mesor of Per1 was significantly higher in $\mathrm{CCl}_{4}$-treated mice. While Per 1 mRNA levels dramatically elevated, the mRNA rhythmicities of other clock genes were maintained and significantly declined in $\mathrm{CCl}_{4}$-treated mice
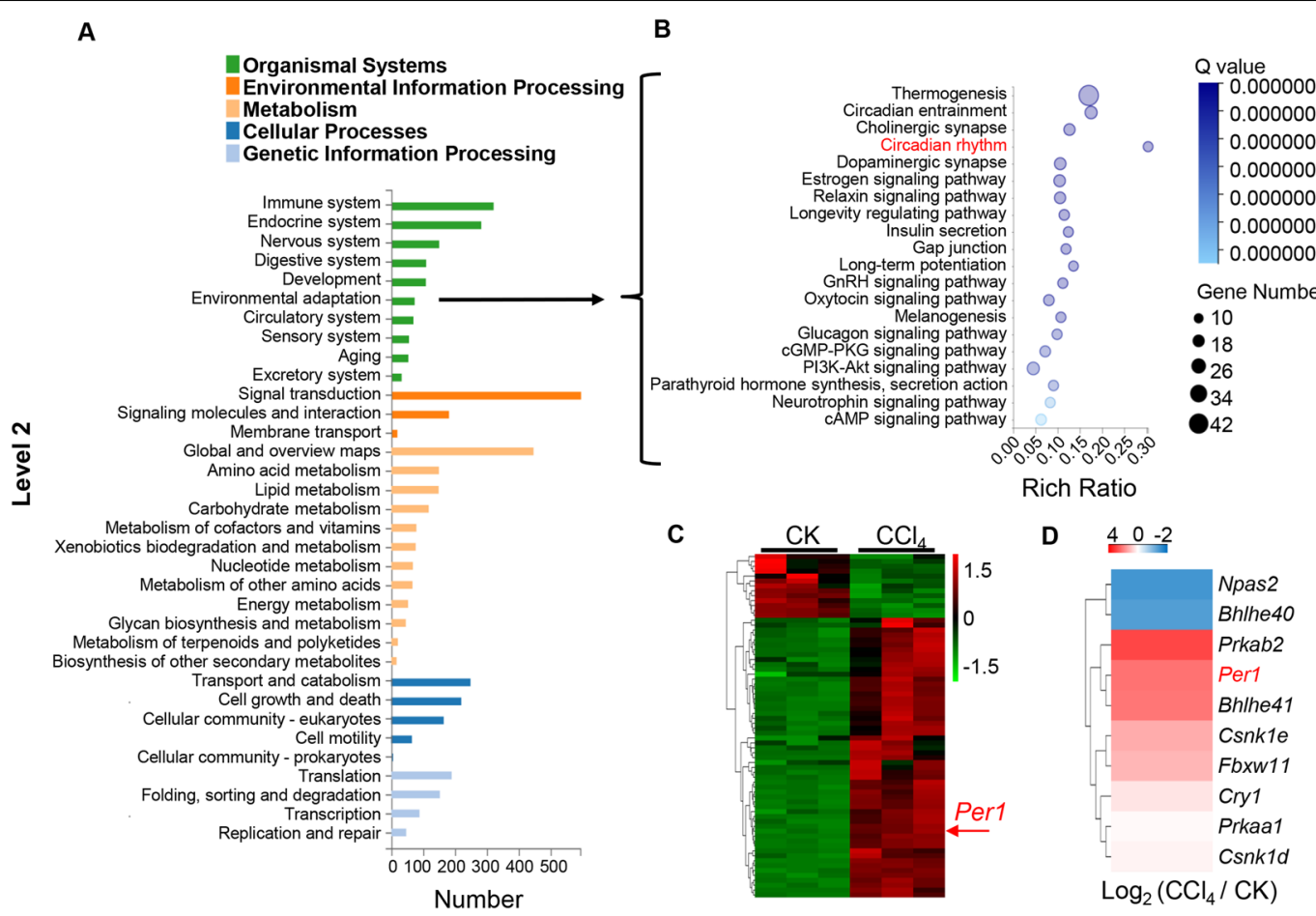

Fig. 1 Per 1 mRNA levels were upregulated significantly in mice exposed to carbon tetrachloride identified by RNA-Seq analysis. Liver tissues were collected $24 \mathrm{~h}$ after a single $\mathrm{CCl}_{4}$ treatment. Samples were analyzed by RNA-seq and differentially expressed pathways. A Distribution of the level 2 KEGG pathways in both $\mathrm{CK}_{\text {and }} \mathrm{CCl}_{4}$. The bar chart showed the numbers of sequences that were assigned within different pathway categories. B Top 20 pathways in the environmental adaptation from A were shown. C Heat map of 70 DEGs in the environmental adaptation pathway from $\mathbf{A}$. D DEGs in the Circadian rhythm pathway from $\mathbf{B}$ were shown. CK: control. $N=3$ independent biological replicates per group. 

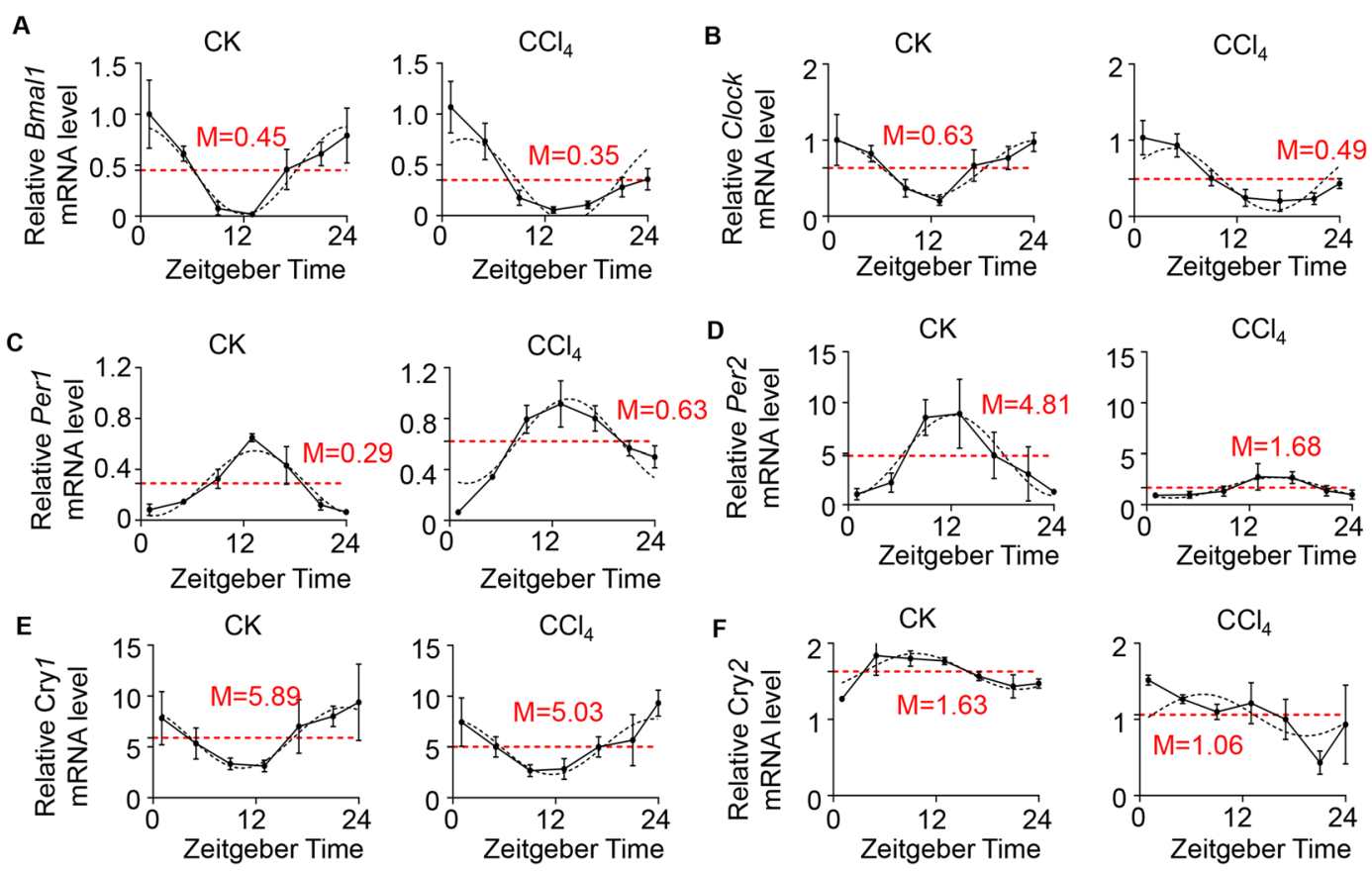

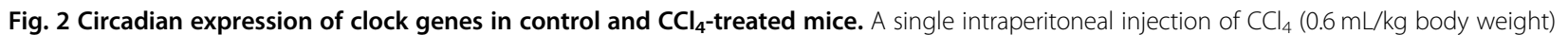
was administrated to WT at ZT1. Mice were killed at ZT1, ZT5, ZT9, ZT13, ZT17, ZT21, and ZT24. Hepatic mRNA levels of Bmal1 A, Clock B, Per1 C, Per2 D, $\mathrm{Cry} 1 \mathbf{E}$, and $\mathrm{Cry} 2 \mathbf{F}$ were measured by real-time PCR after $\mathrm{CCl}_{4}$ treatment. CK: control. Solid lines indicate experimental response curves; dashed lines, fitted model curves. Red dashed line with the number represents mesor. Data were shown as means \pm S.E.M. $N=5$ independent biological replicates per group. Data represent cumulative results from three independent experiments.

Table 1 Circadian rhythmic parameters of clock gene transcriptions in control and $\mathrm{CCl}_{4}$-treated mice.

\begin{tabular}{llll}
\hline Gene & Mesor & Amplitude & Acrophase ZT (h) \\
\hline Control & & & \\
Bmal1 & $0.45 \pm 0.09$ & $0.43 \pm 0.12$ & $0.08 \pm 1.22$ \\
Clock & $0.63 \pm 0.08$ & $0.36 \pm 0.11$ & $0.27 \pm 1.33$ \\
Per1 & $0.29 \pm 0.04$ & $0.25 \pm 0.05$ & $13.33 \pm 0.88$ \\
Per2 & $4.81 \pm 0.93$ & $3.95 \pm 1.24$ & $12.23 \pm 1.34$ \\
Cry1 & $5.89 \pm 0.92$ & $2.94 \pm 1.24$ & $22.36 \pm 1.83$ \\
Cry2 & $1.63 \pm 0.08$ & $0.23 \pm 0.10$ & $9.63 \pm 1.85$ \\
CCl & & & \\
Bmal1 & $0.35 \pm 0.11$ & $0.40 \pm 0.15$ & $2.71 \pm 1.49$ \\
Clock & $0.49 \pm 0.10$ & $0.41 \pm 0.15$ & $4.28 \pm 1.22$ \\
Per1 & $0.63 \pm 0.08^{* *}$ & $0.33 \pm 0.10$ & $13.95 \pm 1.15$ \\
Per2 & $1.68 \pm 0.29^{*}$ & $1.00 \pm 0.40$ & $15.10 \pm 1.49$ \\
Cry1 & $5.03 \pm 0.78$ & $2.73 \pm 1.02$ & $23.58 \pm 1.64$ \\
Cry2 & $1.06 \pm 0.17^{*}$ & $0.27 \pm 0.24$ & $7.38 \pm 4.28$ \\
\hline
\end{tabular}

Data represent means \pm S.E.M., $* P<0.05, * * P<0.01$ vs. control group.

livers (Fig. 2A-F). These findings revealed that $\mathrm{CCl}_{4}$ induced liver injury resulted in changes in the expression of circadian clock genes with a specific elevation of Per1 mRNA. Therefore, these observations raised a fundamental question to the biological relevance for such gene regulations.
Per1 deficiency decreased the metabolism rate of toxins

In order to show that the specific expression of Per1 caused by toxins is related to toxin degradation, a biochemical assay was undertaken to measure $\mathrm{CCl}_{4}$ residual levels in the plasma of WT and Per1 knockout $\left(\operatorname{Per}^{-1-}\right)$ mice after given a single intraperitoneal injection of $\mathrm{CCl}_{4}$. Plasma extract obtained from $\mathrm{CCl}_{4}$-treated $\mathrm{Per}^{-1-}$ mice displayed a higher level of $\mathrm{CCl}_{4}$ residue compared with that of WT mice, indicating loss of Per1 decreased liver metabolism rate of $\mathrm{CCl}_{4}$ (Fig. 3a). Next, we investigated whether the metabolism of other toxins was slowed down in $\operatorname{Per}^{-1-}$ mice. Certain concentrations capsaicin and APAP were intragastrically or intraperitoneally administrated into two genotypes of mice, respectively. The results showed that the residues of capsaicin in the plasma of $\operatorname{Per} 1^{-1-}$ mice were significantly higher than those in wild-type mice, either in the morning or at night (Fig. S1A). Moreover, more APAP residues in plasma were observed after the APAP intraperitoneal administration in Per1 $1^{-1-}$ mouse (Fig. S1B). Together, these results indicated that Per1 deficiency reduced the metabolic rate of toxins.

Per1 deficiency decreased $\mathrm{CCl}_{4}$-induced acute liver injury

Then, we found an alleviated liver injury in $\mathrm{Per}^{-1-}$ mice, reflecting with significantly decreased AST and ALT 


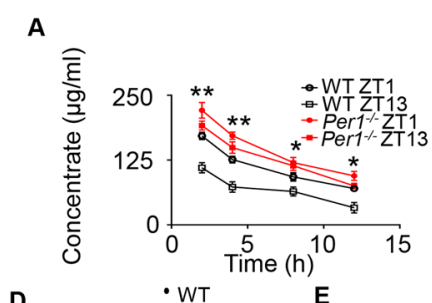

B

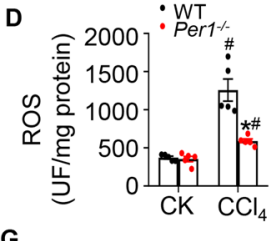

E
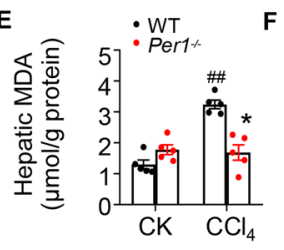

Per1 $\% \quad \mathrm{WT}+\mathrm{CCl}_{4}$

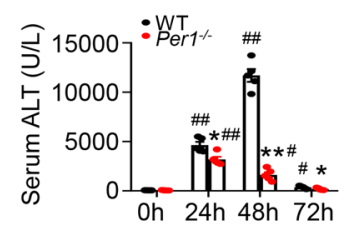

$\mathbf{F}$
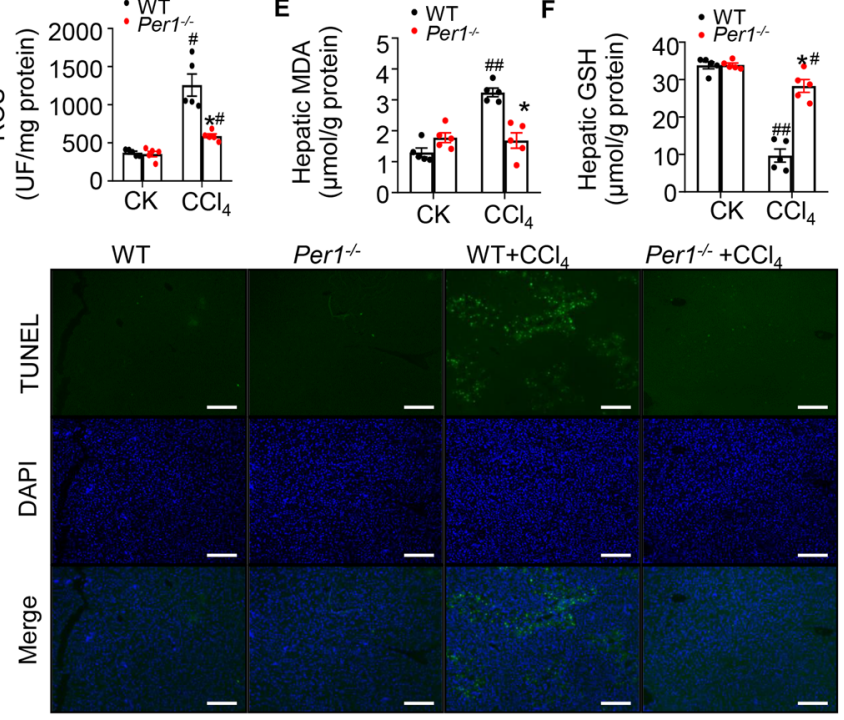

C

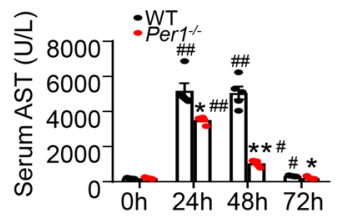

H

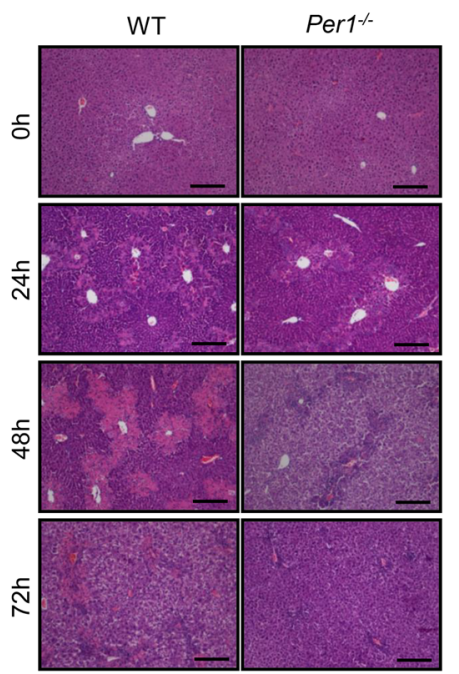

Fig. 3 Effect of Per1 deficiency on $\mathbf{C C l} 4$-induced acute liver injury in mice. A The time course of plasma $\mathrm{CCl}_{4} \mathrm{Concentrations} \mathrm{from} 0$ to $12 \mathrm{~h}$ following a single intraperitoneal injection of $\mathrm{CCl}_{4}(0.6 \mathrm{~mL} / \mathrm{kg}$ body weight). B Serum activities of $\mathrm{ALT}$ and $\mathbf{C}$ AST at 24,48 , and $72 \mathrm{~h}$ after single CCl 4 treatment were measured. Liver tissues were collected 24,48 , and $72 \mathrm{~h}$ after single $\mathrm{CCl}_{4}$ treatment. Hepatic concentrations of $\mathbf{D}$ ROS production, E MDA, and $\mathbf{F}$ GSH were measured $24 \mathrm{~h}$ after single $\mathrm{CCl}_{4}$ treatment. G Representative immunostaining images. Apoptotic cells were visualized by TUNEL staining (green) and counterstained with DAPI (blue). $\mathbf{H}$ Representative H\&E staining of livers from each treatment group. CK: control.

Bar $=100 \mu \mathrm{m}$. Representative images from $\mathrm{N}=3$ biological replicates. Data were shown as means \pm S.E.M. $N=5$ independent biological replicates per group. ${ }^{*}$ indicates $P$ value $<0.05,{ }^{* *}$ indicates $P$ value $<0.01$, Per $1^{-1}$ group versus WT group; ${ }^{\#}$ indicates $P$ value $<0.05$, ${ }^{\# \#}$ indicates $P$ value $<0.01$, $\mathrm{CCl}_{4}$ group versus control group. Data represent cumulative results from three independent experiments.

levels at 24, 48, and $72 \mathrm{~h}$ after $\mathrm{CCl}_{4}$ administration (Fig. $3 \mathrm{~B}, \mathrm{C})$. The levels of hepatic ROS were also significantly lower in Per1 ${ }^{-1-}$ mice compared with WT mice (Fig. 3D), but the MDA levels did not significantly change after $\mathrm{CCl}_{4}$ (Fig. 3E). The hepatic GSH level was reduced by $\mathrm{CCl}_{4}$ administration, with a further reduction in WT mice compared with Per1 $^{-1-}$ mice (Fig. 3F). Apoptotic cells were visualized by TUNEL staining and counterstained with DAPI. Treatment with $\mathrm{CCl}_{4}(24 \mathrm{~h})$ induced a significant apoptotic response, consistent with the above results (Fig. 3G). Apoptosis was significantly less frequent in the liver of $\operatorname{Per}^{-1-}$ mice as compared with WT mice, as seen by TUNEL (Fig. 3G). Histological examination of the liver tissues revealed that hepatic damage was limited to pericentral areas in mice at $24 \mathrm{~h}$ (Fig. $3 \mathrm{H})$. The necrosis was less severe in the $\operatorname{Per1}^{-1-}$ mice compared with WT mice during 24-72 $\mathrm{h}$ after $\mathrm{CCl}_{4}$ administration. These observations suggested that the elevation of Per1 expression in toxin $\mathrm{CCl}_{4}$ exposure was associated with the enhancement of liver metabolism of toxins capability.

\section{Per1 deficiency alleviated chronic $\mathrm{CCl}_{4}$-induced liver injury} and fibrosis in mice

To expand our observation that loss of Per1 reduced $\mathrm{CCl}_{4}$-induced liver acute injury, we performed a series of experiments to assess $\mathrm{CCl}_{4}$-induced chronic liver injury and fibrosis in WT and Per1 ${ }^{-1-}$ mice. Mice were injected with $0.6 \mathrm{~mL} / \mathrm{kg}$ body weight $\mathrm{CCl}_{4}$ twice weekly for 4 weeks and killed $48 \mathrm{~h}$ after the last injection. As expected, histological examination of the liver tissues after $\mathrm{CCl}_{4}$ administration also revealed significantly less severe necrosis in Per1 $^{-1-}$ mice compared with WT mice (Fig. 4A, B). Chronic liver injury was alleviated in $\mathrm{Per}^{-1-}$ mice as evidenced by lower serum ALT and AST levels after $\mathrm{CCl}_{4}$ administration (Fig. 4G, H). After chronic treatment of $\mathrm{CCl}_{4}$ for 4 weeks, WT mice had significant hepatic fibrosis, as demonstrated by Masson's trichrome staining. In contrast, $\mathrm{Per}^{-1-}$ mice had less fibrosis as demonstrated by reduced collagen deposition (Fig. 4C, D). Expression of $\alpha$-SMA, a marker of hepatic stellate cell (HSC) activation, was increased in both genotypes in 


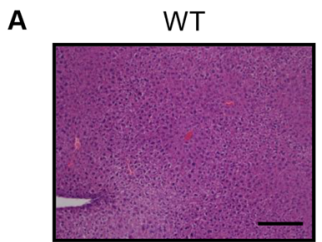

C

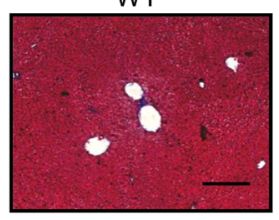

E
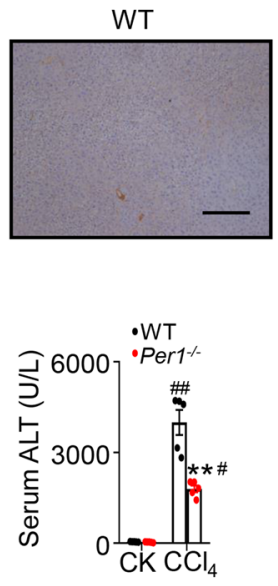

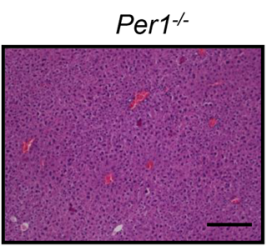

Per1 $1-$

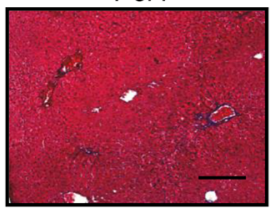

Per1\%

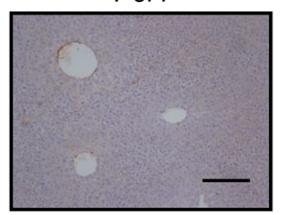

H

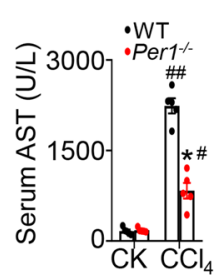

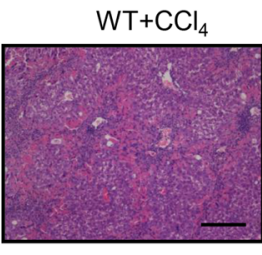

$\mathrm{WT}+\mathrm{CCl}_{4}$

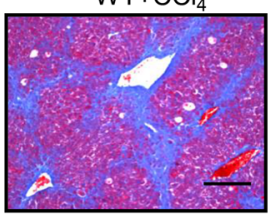

$\mathrm{WT}+\mathrm{CCl}_{4}$
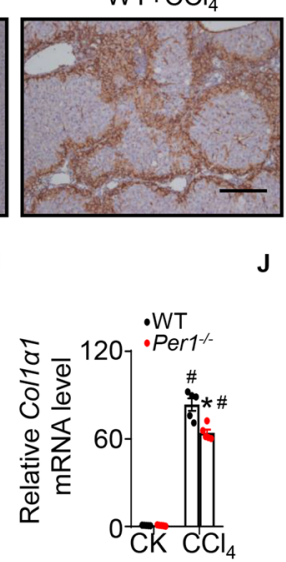

J
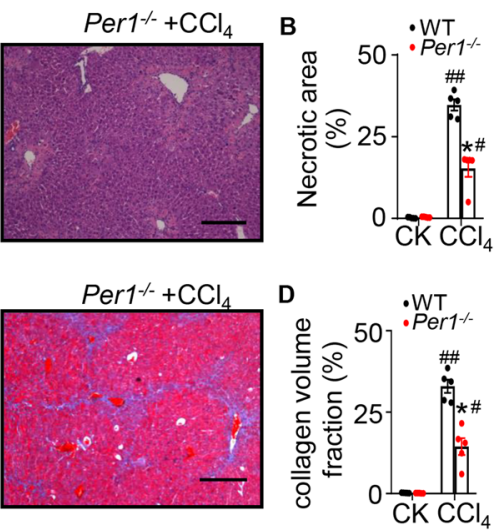

Per1 $\%+\mathrm{CCl}_{4}$
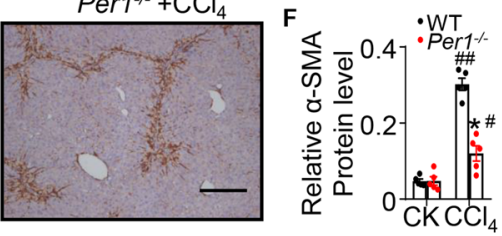

K
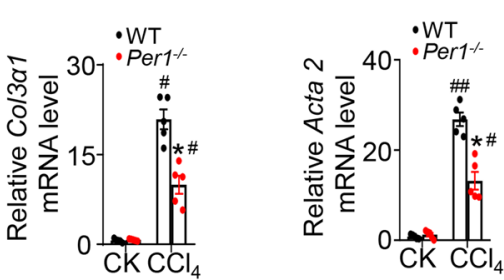

Fig. 4 Effect of Per1 deficiency on liver injury induced by chronic $\mathrm{CCl} 4$ administration. A H\&E staining of livers from each treatment group. B The percentage of necrotic area was quantified using Image J software. C Masson's trichrome of livers from each treatment group for the visualization of hepatic collagen deposition. D The percentage of collagen volume fraction was quantified using Image J software. E Expression of a-SMA was determined by immunohistochemistry. F Relative a-SMA Protein level was quantified using Image J software. Bar $=100 \mu \mathrm{m}$. Representative images from $N=3$ biological replicates. Serum activities of $\mathbf{G}$ ALT and $\mathbf{H}$ AST were measured after chronic $\mathrm{CCl}_{4}$ administration. Relative mRNA levels of $\mathbf{I}$ Colla 1 and $\mathbf{J}$ Col3a1 were measured in the liver of WT and Per1 ${ }^{-1-}$ mice. $\mathbf{K}$ Relative mRNA levels of Atca2 were measured in liver of WT and Per1 ${ }^{-1-}$ mice. CK: control. Data were shown as means \pm S.E.M. $N=5$ independent biological replicates per group. ${ }^{*}$ indicates $P$ value $<0.05,{ }^{* *}$ indicates $P$ value $<0.01$, Per $^{-1-}$ group versus WT group; ${ }^{\#}$ indicates $P$ value $<0.05,{ }^{\# \#}$ indicates $P$ value $<0.01, C C C_{4}$ group versus control group.

response to chronic $\mathrm{CCl}_{4}$ treatment. Perl deficiency significantly reduced $\alpha$-SMA expression after $\mathrm{CCl}_{4}$. administration as assessed by immunohistochemistry (Fig. 4E, F). $\mathrm{CCl}_{4}$ administration elevated mRNA levels of markers for fibrogenesis including Col1 $\alpha 1, \operatorname{Col} 3 \alpha 1$, and Acta2. The expression of all these genes after $\mathrm{CCl}_{4}$ treatment was significantly suppressed in $\operatorname{Per} 1^{-1-}$ mice compared with WT mice (Fig. 4I-K). These results indicated that $\operatorname{Per} 1$ deficiency alleviated chronic $\mathrm{CCl}_{4}$-induced liver injury and fibrosis in mice.

\section{Per1 deficiency declined the level of Cyp2e1 expression in vivo}

As circadian genes can influence hepatic oxidative stress by mediating the expression of antioxidative enzymes in mice liver ${ }^{27,28}$, we examined the effect of Per1 deletion on hepatic expression of these enzymes, including Gclc, Ho-1,
Cat, Sod1, and Sod2. $\mathrm{CCl}_{4}$ treatment significantly elevated the expression of Gclc and Ho-1. Cat, Sod1, and Sod2 were downregulated in response to $\mathrm{CCl}_{4}$. These antioxidative enzymes showed no significant changes between WT and Per $1^{-1-}$ mice (Fig. 5A-E). The injurious effects of a few toxins are results of their intermediary metabolites by CYP2E1, some highly toxic molecules that can lead to oxidative injury ${ }^{29,30} \cdot \mathrm{CCl}_{4}$ is metabolized by CYP2E1 to trichloromethyl peroxyl radical that induces oxidative stress cell damage and cell death ${ }^{31}$. The change of Cyp $2 e 1$ expression by qRT-PCR analysis was in agreement with RNA-seq data (Fig. S2). Intraperitoneal injection of mice with $\mathrm{CCl}_{4}$ decreased Cyp2e1 expression as previously described $^{31}$. Analysis of mRNA levels in the liver revealed that Cyp2e1 expression was significantly downregulated by Per 1 deletion in both control and $\mathrm{CCl}_{4}$ group (Fig. $5 \mathrm{~F}$ ), indicating a possibility of decreased hepatic injury after 

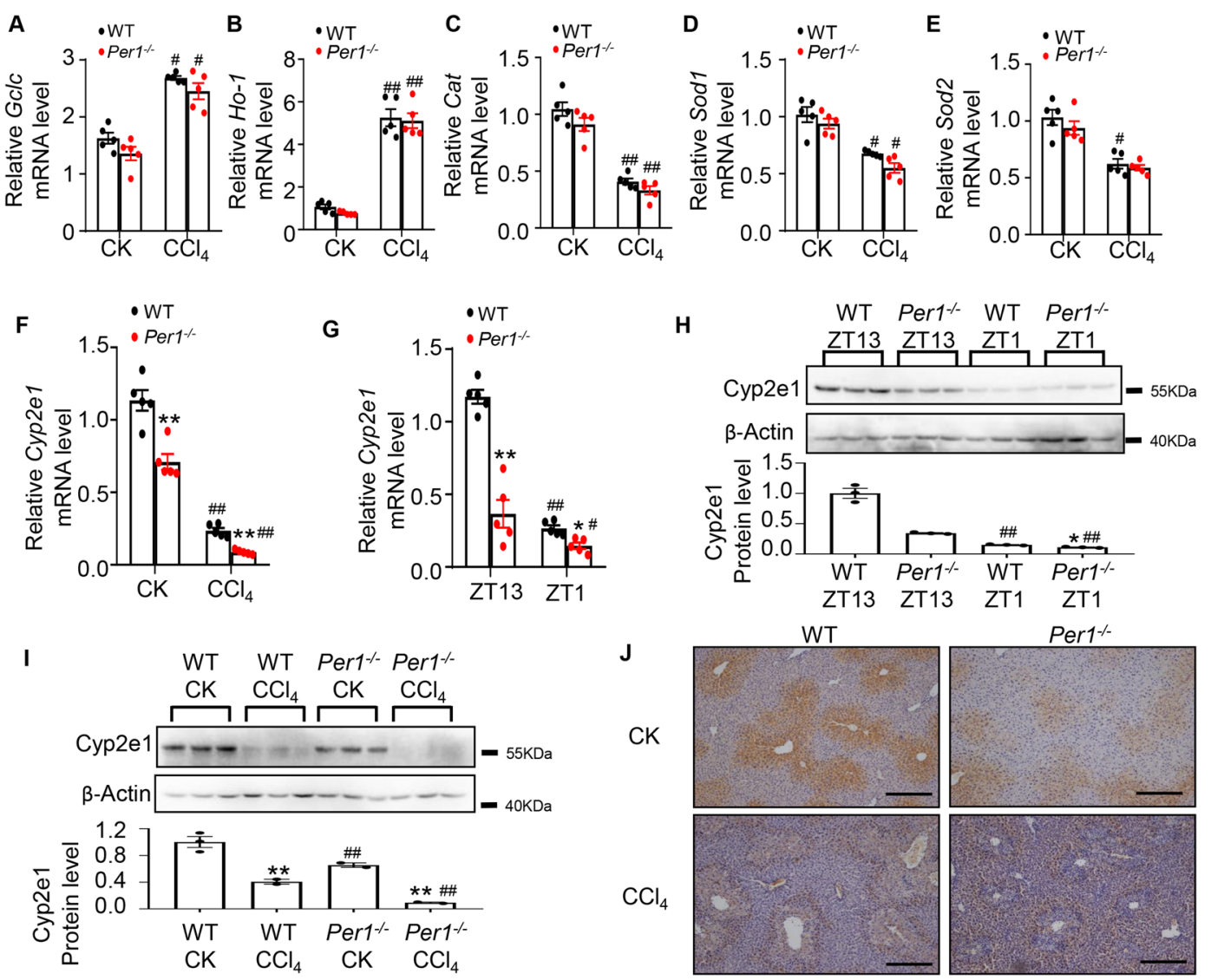

Fig. 5 Effect of Per1 deficiency on Cyp2e1 expression exposed to CCl4. Liver tissues were collected $24 \mathrm{~h}$ after single $\mathrm{CCl}_{4}$ administration. Hepatic mRNA levels of $\mathbf{A}$ Gclc, B Ho-1, C Cat, D Sod1, E Sod2, and $\mathbf{F}$ Cyp2el were measured by real-time PCR. CK: control. Data were shown as means \pm S.E.M. $N=5$ independent biological replicates per group. ${ }^{*}$ indicates $P$ value $<0.05,{ }^{* *}$ indicates $P$ value $<0.01, P e r 1^{-/}$group versus WT group; ${ }^{*}$ indicates $P$ value $<0.05,{ }^{\# \#}$ indicates $P$ value $<0.01, C^{C C l}$ group versus control group. $\mathbf{G}$ Cypzel expression was measured by real-time PCR in the mouse liver at ZT1 and ZT13. H Representative western blots for rhythm of CYP2E1 protein expression in the mouse liver. Extracts were measured via western blot analysis with anti-CYP2E1 or anti- $\beta$-ACTIN antibody. CK: control. Data were shown as means \pm S.E.M. $N=3$ independent biological replicates per group. ${ }^{*}$ indicates $P$ value $<0.05,{ }^{* *}$ indicates $P$ value $<0.01, P e r 1^{-1-}$ group versus WT group; ${ }^{\#}$ indicates $P$ value $<0.05$, \#\# indicates $P$ value $<0.01, Z T 1$ group versus ZT13 group. I Western blot assay of CYP2E1 protein after $\mathrm{CCl}_{4}$ treatment in vivo. $\mathbf{J}$ Representative images of immunohistochemical staining were shown for liver sections stained with anti-CYP2E1 antibody. This is in agreement with the RT-PCR and western blot data. Bar $=100 \mu \mathrm{m}$. Representative images from $N=3$ biological replicates. CK: control. Data were shown as means \pm S.E.M. $N=3$ independent biological replicates per group. ${ }^{*}$ indicates $P$ value $<0.05$, ${ }^{* *}$ indicates $P$ value $<0.01, P e r 1^{-1-}$ group versus WT group; ${ }^{\#}$ indicates $P$ value $<0.05$, ${ }^{\# \#}$ indicates $P$ value $<0.01, C C l_{4}$ group versus control group. Data represent cumulative results from three independent experiments.

$\mathrm{CCl}_{4}$ was primarily owing to downregulation of Cyp2e1 in $\operatorname{Per1}^{-1-}$ mice. Then we investigated whether the expression of Cyp2e1 was circadian controlled. The results showed the mRNA expression of Cyp2e1 was maximal at the beginning of the dark phase of the entraining photocycle at ZT13 and minimal at ZT1, the onset of the light phase (Fig. 5G). The knockdown of Per1 resulted in an approximately twofold diminution of Cyp2e1 expression at the mRNA level (Fig. 5G). Western blot analysis revealed that CYP2E1 protein levels were higher during the dark phase (Fig. 5H). The CYP2E1 protein was also reduced in $\operatorname{Per} 1^{-1-}$ mice, and the circadian rhythm of CYP2E1 was impaired (Fig. 5H).
Analysis of protein levels in the liver revealed that CYP2E1 expression was significantly downregulated by Per1 deletion in both control and $\mathrm{CCl}_{4}$ group (Fig. 5I). Next, we performed immunoblotting analysis to examine putative changes in CYP2E1 protein levels in mouse liver tissues, resulting from $\mathrm{CCl}_{4}$ treatment. Parallel immunohistochemical examination revealed that CYP2E1positive staining was decreased in $\mathrm{Perl}^{-1-}$ mice exposed $\mathrm{CCl}_{4}$ compared with WT mice (Fig. 5J). Taken together, these findings demonstrated that Per1 participated in the regulation of transcriptional activation of Cyp2e1 and, in turn, played a critical role in mediating $\mathrm{CCl}_{4}$-induced hepatotoxicity. 


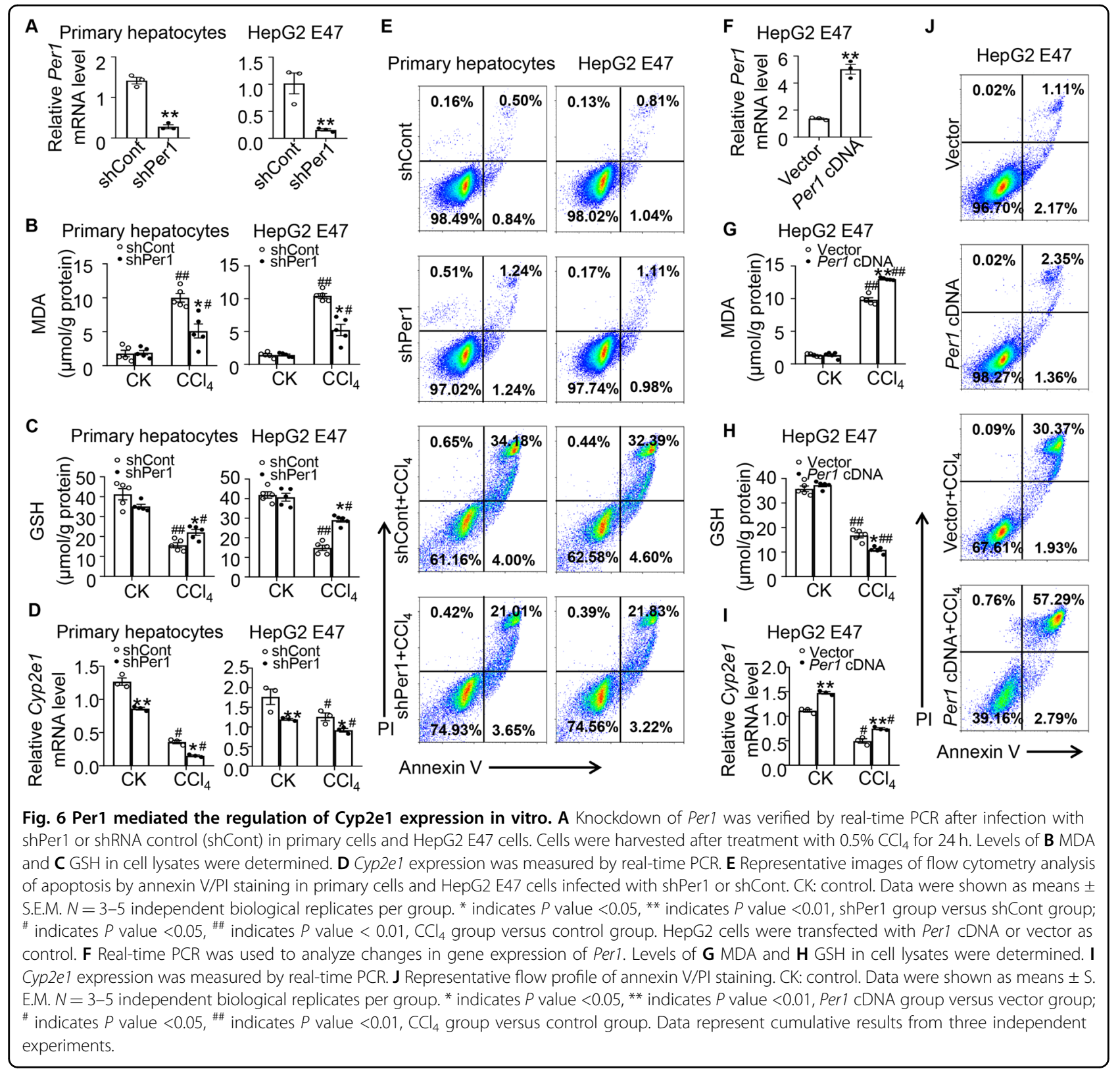

\section{Effect of Per1 on $\mathrm{CCl}_{4}$-induced oxidative injury and Cyp2e1 expression in hepatocytes}

To investigate whether Per1 directly regulated Cyp $2 e 1$ expression in hepatocytes, we analyzed $\mathrm{CCl}_{4}$-induced oxidative injury in primary hepatocytes and HepG2 E47 cells. The primary hepatocytes were transfected with control shRNA or Per1-specific shRNA, and the cells were challenged with $\mathrm{CCl}_{4}$. shRNA-mediated knockdown of Per1 led to a 70-80\% decrease in mRNA levels (Fig. 6A). Next, we created stable HepG2 E47 cell lines expressing shPer1 through retroviral infection and assessed the effect of Per1 suppression on the expression of Cyp2e1. As expected, real-time PCR experiments showed that cells treated with Per1 shRNA decreased the expression level of Per1 (Fig. 6A). $\mathrm{CCl}_{4}$ exposure increased intracellular MDA levels, and suppression of Per1 expression significantly impaired the elevation of MDA in primary hepatocytes (Fig. 6B). The contents of GSH in cells also decreased after $\mathrm{CCl}_{4}$, which could be alleviated by knockdown of Per 1 in primary hepatocytes (Fig. 6C). Per1 inhibition improved the increase in MDA levels and the decrease in GSH levels in HepG2 E47 cells (Fig. 6B, C). Per1 inhibition also effectively reduced mRNA levels of Cyp2e1 in primary hepatocytes (Fig. 6D). Per1 inhibition reduced Cyp2e1 mRNA levels in HepG2 E47 cells, consistent with the results of primary hepatocytes (Fig. 6D). Apoptosis was 
measured by annexin V/PI staining followed by flow cytometry. Compared with control shRNA, the cells treated with Per1 shRNA showed less apoptosis in vitro in primary cells (Fig. 6E). Consistent with the above observation, inhibition of Per1 expression resulted in the reduction of apoptosis in HepG2 E47 cells (Fig. 6E). To further study the function of Per1, HepG2 E47 cells expressing Cyp2e1 were transfected with Per1-cDNA plasmid and then treated with $\mathrm{CCl}_{4}$ for $24 \mathrm{~h}$. Overexpression was verified by real-time PCR (Fig. 6F). Overexpression of Per1 in HepG2 E47 increased $\mathrm{CCl}_{4}$ toxicity. Moreover, MDA induced by $\mathrm{CCl}_{4}$ was increased to a greater extent, and GSH was further decreased (Fig. 6G, $\mathrm{H})$.The Per1 transfection significantly increased Cyp $2 e 1$ expression in hepatocytes both in control and $\mathrm{CCl}_{4}$ group (Fig. 6I), which was consistent with that observed in vivo. We observed a significant increase in apoptosis after $\mathrm{CCl}_{4}$ in cells of Per1 cDNA transfected (Fig. 6J).

\section{The Per1 increased Cyp2e1 expression by interacting with HNF-1a on Cyp2e1 promoter}

Next, we investigated how Per1 regulates Cyp2e1 expression. Cyp2e1 exhibits 24-hour periodicity in its hepatic mRNA levels, and this circadian rhythm is regulated by HNF- $1 \alpha$ and the circadian organization of molecular clocks ${ }^{12}$. Both hepatic mRNA and protein levels of HNF-1 $\alpha$ were similar between WT and Per1 $^{-1-}$ mice (Fig. 7A-C). The role of PER1 in the transcriptional activation of HNF- $1 \alpha$ was confirmed by ChIP-qPCR, which showed a marked reduction (about twofold) in HNF-1 $\alpha$ binding to Cyp2e1 promoter regions in Per1-deficient liver tissues (Fig. 7D, E). PER1 protein also occurred in the HNF-1 $\alpha$ binding site in the Cyp2e1 promoter (Fig. 7F), implying a possible interaction between PER1 and HNF-1 $\alpha$. Coimmunoprecipitation and western blot analysis of the liver lysates showed that HNF-1 $\alpha$ interacted with PER1 and forms a protein complex (Fig. 7G). Interaction of CREB binding protein $(\mathrm{CBP})$ with the $\mathrm{N}$-terminal domain of HNF-1 greatly increased the binding affinity ${ }^{32}$. To further verify the specificity of HNF-1 $\alpha$ to the PER1, we tested the binding of CBP to PER1. CBP was also coimmunoprecipitated by the anti-PER1 (Fig. 7G). Not surprisingly, we also observed strongly synergy between PER1 and HNF-1 $\alpha$, whereas CBP specifically interacted with HNF- $1 \alpha$ in the whole-liver homogenates immunoprecipitated with anti-HNF-1 $\alpha$ antibody (Fig. 7H). These results indicated that PER1 protein interacted with the HNF$1 \alpha-\mathrm{CBP}$ complex and increased the binding activity of HNF-1 $\alpha$ on the Cyp2e1 promoter, enhancing the transcription of Cyp2e1 in mice liver.

\section{Discussion}

Our observations that toxins specifically activate Per1 expression illustrate the central role played by the circadian clock in mediating the metabolism of toxins physiology in mice. Our previous study showed that Per2 plays a protective role in $\mathrm{CCl}_{4}$-induced hepatotoxicity ${ }^{33}$. The opposite gene expression of Per1 and Per2 in mice exposed to $\mathrm{CCl}_{4}$ suggested different roles of core circadian genes. Per2 gene functions in hepatocyte protection from chemical toxicants via the regulation of hepatic Ucp 2 gene expression levels ${ }^{33}$. Loss of Per1 reduced metabolism of toxins capability, and eliminated the difference of toxin residues in plasma between day and night, suggesting Per1 is required for maintaining daily xenobiotic-metabolizing rhythms. It is well known that Cyp $2 e 1$ is responsible for the metabolism of most xenobiotics and is responsible for the efficient elimination of foreign chemicals from the body ${ }^{34}$. Our results supported that the metabolizing capacity of Per1 is through regulating Cyp $2 e 1$ expression. Paradoxically, Cyp $2 e 1$ metabolically activates biologically inert compounds to electrophilic derivatives that can cause toxicity and cell death ${ }^{10,11}$. CYP2E1 catalyzes $\mathrm{CCl}_{4}$ molecule to the trichloromethyl free radical and induces hepatotoxicity. Elevation of CYP2E1 activity with phenobarbital, dichlorodiphenyltrichloroethane, or Arochlor $1254 \mathrm{R}$ increases the hepatotoxic effect of $\mathrm{CCl}_{4}{ }^{35}$. Mice lacking Cyp $2 e 1$ are resistant to $\mathrm{CCl}_{4}$ hepatotoxicity ${ }^{36}$. Therefore, it is reasonable that mice lacking Per1 alleviate $\mathrm{CCl}_{4}$ induced liver injury and fibrosis due to the low level of hepatic Cyp2e1.

The Cyp2e1 expression and activities are circadian controlled with higher levels in the dark phase in mice liver ${ }^{12,37}$, and this rhythm was impaired in $P e r 1^{-/-}$mice. Previous study has demonstrated transcription of the Cyp $2 e 1$ is rhythmically enhanced by HNF- $1 \alpha$, and circadian gene CRY1 rhythmically inhibits the binding activity of HNF-1 $\alpha$ on Cyp $2 e 1$ promoter $^{12,38,39}$. PER and CRY play a central role in negative-feedback loop in the core clock system. The circadian pattern of Per1 mRNA in the liver is consistent with the rhythmic expression of Cyp2e1, which peaks at ZT12 as previously described ${ }^{40}$. These evidences implied a possible role of Per1 in regulating the circadian transcription of Cyp2e1. In the present study, we showed that Per1 directly enhanced mRNA levels of Cyp2e1. The expression of HNF-1 $\alpha$ was not affected by Per1. However, the recruitment of HNF-1 $\alpha$ to Cyp $2 e 1$ promoter was significantly weakened in $\mathrm{Per1}^{-1-}$ mice. ChIP assays also revealed the recruitment of PER1 protein to the binding site of HNF- $1 \alpha$ in the Cyp $2 e 1$ promoter. Previous study of the crystal structure of PER1 protein demonstrated that PER1 cannot directly bind to DNA ${ }^{41}$, implying that PER1 may associate with other proteins and indirectly bind to the Cyp2e1 promoter. Results of coimmunoprecipitated assays revealed a novel interaction between PER1 and HNF-1 $\alpha$. The present study suggests that the Per1 maintains the daily metabolism of toxin 
A

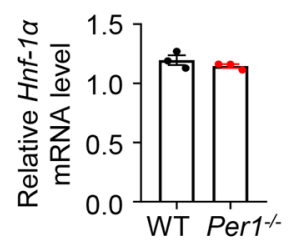

B

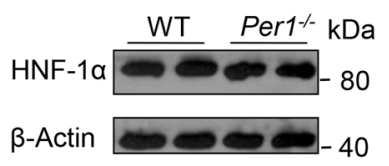

C

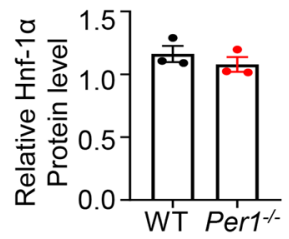

$\mathbf{F}$

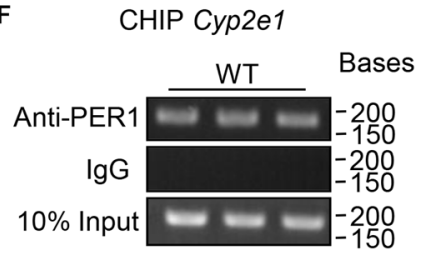

G

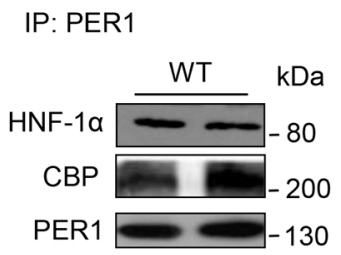

H

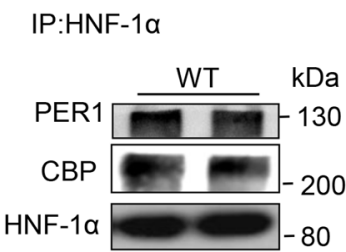

E CHIP-qPCR

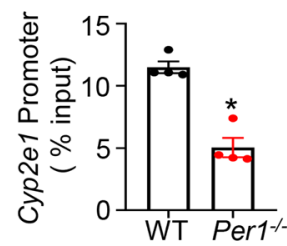

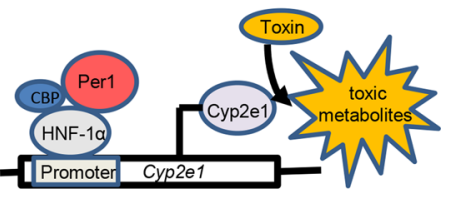

Fig. 7 PER1 interacted with HNF-1a on Cyp2e1 promoter. A Hepatic mRNA levels of HNF-1a were measured in Per1 ${ }^{-1-}$ and WT mice. B-C Hepatic protein levels of HNF-1a were analyzed by western blot and $\beta$-actin was used as internal control. D Recruitments of HNF-1a to Cyp2e1 promoter were detected by ChIP assays. E ChIP assays were performed followed by real-time PCR. F Recruitments of PER1 to Cyp2e1 promoters were detected by ChIP assays. G-H Representative immunoblots showing recovery of the indicated proteins from mouse liver after immunoprecipitation with the indicated antibodies. I A hypothetical model of how PER1 interacted with HNF-1a on Cyp2el promoter and target gene transcription. Data were shown as means \pm S.E.M. $N=5$ independent biological replicates per group; ${ }^{*} P<0.05$, Per $^{-/-}$group versus WT group.

rhythm through regulation of Cyp2e1 expression (Fig. 7I). Our findings presented a control system that causes alternating turn-off/turn-on of transcription on Cyp $2 e 1$ by endogenous circadian PER1 interaction.

The overarching evidences have already showed a reciprocal regulation of circadian rhythms and metabolic states. Disrupted rhythms can lead to metabolic impairments, the reverse is also true that disrupting metabolism can alter circadian rhythms ${ }^{3}$. Patients with cirrhosis have liver portal hypertension and dysfunctional circadian clock systems ${ }^{5}$. Hepatic fibrosis induced by $\mathrm{CCl}_{4}$ leads to alterations in the rhythms of hepatic clock genes ${ }^{6}$, and the patients with hepatic fibrosis suffer abnormal sleeping cycles $^{42}$, indicating possible side-effects of clock responding to liver injury and metabolic diseases. Per1 is the unique clock gene that responds rapidly to toxin exposure, and this response inevitably influences the normal circadian system.

There are multiple CRE binding sites in the promoter of Per1 gene, which may be caused by cAMP signal (enriched in KEGG). Loss of Per1 reduced significantly $\mathrm{CCl}_{4}$ induced acute and chronic liver injury. Thus, appropriate suppression of Per1 response is a potential therapeutic target for toxin-induced hepatotoxicity, also is a promising method to improve the dysfunctional circadian clock system caused by toxin metabolism.

\section{Acknowledgements}

This work was supported by the grant from the National Natural Science Foundation of China $(31671220,31871178)$ and the Fundamental Research Funds for the Central Universities (30920031102).

\section{Author contributions}

W.H.G. and T.W. performed the experiments. Y.Z., Y.X.Y., Q.S., X.Y., G.Y., and X.X. contributed reagents and analytical tools. J.F.Z. supervised the project and conceived the study and designed the experiments. W.H.G. and J.F.Z. wrote the manuscript with input from other authors, and all authors revised it.

\section{Data availability}

Data are available upon request from the corresponding author.

\section{Conflict of interest}

The authors declare that they have no conflict of interest.

Ethics approval and consent to participate

All procedures were approved by the Animal Care and Use Committee at Nanjing University of Science and Technology (ACUC-NUST-20160016).

\section{Publisher's note}

Springer Nature remains neutral with regard to jurisdictional claims in published maps and institutional affiliations. 
Supplementary Information accompanies this paper at (https://doi.org/ 10.1038/s41419-020-03343-7).

Received: 2 November 2020 Revised: 7 December 2020 Accepted: 10 December 2020

Published online: 12 January 2021

\section{References}

1. Reppert, S. M. \& Weaver, D. R. Coordination of circadian timing in mammals. Nature 418, 935-941 (2002).

2. Fu, L., Patel, M. S., Bradley, A., Wagner, E. F. \& Karsenty, G. The molecular clock mediates leptin-regulated bone formation. Cell 122, 803-815 (2005).

3. Arble, D. M., Ramsey, K. M., Bass, J. \& Turek, F. W. Circadian disruption and metabolic disease: findings from animal models. Best. Pract. Res., Clin. Endocrinol. Metab. 24, 785-800 (2010).

4. Tahara, Y. \& Shibata, S. Circadian rhythms of liver physiology and disease: experimental and clinical evidence. Nat. Rev. Gastroenterol. Hepatol. 201613 217-226 (2016).

5. Montagnese, $\mathbf{S}$. et al. Sleep-wake abnormalities in patients with cirrhosis. Hepatology 59, 705-712 (2014).

6. Chen, P., Kakan, X. \& Zhang, J. Altered circadian rhythm of the clock genes in fibrotic livers induced by carbon tetrachloride. FEBS Lett. 584, 1597-1601 (2010).

7. Kleiner, D. E. et al. Hepatic histological findings in suspected drug-induced liver injury: systematic evaluation and clinical associations. Hepatology 59, 661-670 (2014)

8. Malhi, H. \& Gores, G. J. Cellular and molecular mechanisms of liver injury. Gastroenterology 134, 1641-1654 (2008)

9. Bataller, R. \& Brenner, D. A. Liver fibrosis. J. Clin. Invest. 115, 209 (2005).

10. Gong, P. \& Cederbaum, A. I. Nrf2 is increased by CYP2E1 in rodent liver and HepG2 cells and protects against oxidative stress caused by CYP2E1. Hepatology 43, 144-153 (2006).

11. Dey, A., Caro, A. A. \& Cederbaum, A. I. S-adenosyl methionine protects ob/ob mice from CYP2E1-mediated liver injury. Am. J. Physiol. Gastrointest. Liver Physiol. 293, G91-G103 (2007).

12. Matsunaga, N., Ikeda, M., Takiguchi, T., Koyanagi, S. \& Ohdo, S. The molecular mechanism regulating 24-hour rhythm of CYP2E1 expression in the mouse liver. Hepatology 48, 240-251 (2008).

13. Beauchamp, D. \& Labrecque, G. Chronobiology and chronotoxicology of antibiotics and aminoglycosides. Adv. Drug Deliv. Rev. 59, 896-903 (2007).

14. Krista, K. \& Lee, C. C. Reciprocal regulation of haem biosynthesis and the circadian clock in mammals. Nature 430, 467-471 (2004).

15. Hjelle, J. J. \& Petersen, D. R. Decreased in vivo acetaldehyde oxidation and hepatic aldehyde dehydrogenase inhibition in C57BL and DBA mice treated with carbon tetrachloride. Toxicol. Appl. Pharmacol. 59, 15-24 (1981).

16. Cong, $M$. et al. Antifibrotic effects of a recombinant adeno-associated virus carrying small interfering RNA targeting TIMP-1 in rat liver fibrosis. Am. J. Pathol. 182, 1607-1616 (2013).

17. Seglen, P. O. Preparation of isolated rat liver cells. Methods Cell Biol. 13, 29-83 (1976).

18. Zheng, B. et al. Nonredundant roles of the mPer1 and mPer2 genes in the mammalian circadian clock. Cell 105, 683-694 (2001).

19. Kim, D., Langmead, B. \& Salzberg, S. L. HISAT: a fast spliced aligner with low memory requirements. Nat. Methods 12, 357-360 (2015).

20. Tarazona, S., García-Alcalde, F., Dopazo, J., Ferrer, A. \& Conesa, A. Differentia expression in RNA-seq: a matter of depth. Genome Res 21, 2213-2223 (2011).
21. Yang, $\mathrm{P}$. et al. Loss of $\mathrm{A}(1)$ adenosine receptor attenuates alphanaphthylisothiocyanate-induced cholestatic liver injury in mice. Toxicol. Sci. 131, 128-138 (2013).

22. Tang, $\mathrm{X}$. et al. Assessment of Brd4 inhibition in idiopathic pulmonary fibrosis lung fibroblasts and in vivo models of lung fibrosis. Am. J. Pathol. 183, 470-479 (2013).

23. Kong, $X$. et al. Interleukin-22 induces hepatic stellate cell senescence and restricts liver fibrosis in mice. Hepatology 56, 1150-1159 (2012).

24. Zhao, Y. et al. Preparation, characterization, and pharmacokinetics study of capsaicin via hydroxypropyl-beta-cyclodextrin encapsulation. Pharm. Biol. 54, 130-138 (2016).

25. Esteban, A., Graells, M., Satorre, J. \& Pérez-Mateo, M. Determination of paracetamol and its four major metabolites in mouse plasma by reversed-phase ion-pair high-performance liquid chromatography. J. Chromatogr. 573, 121-126 (1992)

26. Mohri, T. et al. Alterations of circadian expressions of clock genes in Dahl saltsensitive rats fed a high-salt diet. Hypertension 42, 189-194 (2003).

27. Hardeland, R., Coto-Montes, A. \& Poeggeler, B. Circadian rhythms, oxidative stress, and antioxidative defense mechanisms. Chronobiol. Int. 20, 921-962 (2003).

28. Xu, Y. Q. et al. Diurnal variation of hepatic antioxidant gene expression in mice. PLoS ONE 7, e44237 (2012).

29. Tsujimoto, I., Moriya, K., Sakai, K., Dickneite, G. \& Saka, T. Critical role of factor XII in the initial stages of carbon tetrachloride-induced adult liver remodeling. Am. J. Pathol. 179, 3011-3019 (2011).

30. Weber, L. W. D., Boll, M. \& Stampfl, A. Hepatotoxicity and mechanism of action of haloalkanes: carbon tetrachloride as a toxicological model. Crit. Rev. Toxicol. 33, 105-136 (2003).

31. Knockaert, L. et al. Carbon tetrachloride-mediated lipid peroxidation induces early mitochondrial alterations in mouse liver. Lab. Invest. 92, 396-410 (2012).

32. Soutoglou, E., Papafotiou, G., Katrakili, N. \& Talianidis, I. Transcriptional activation by hepatocyte nuclear factor-1 requires synergism between multiple coactivator proteins. J. Biol. Chem. 275, 12515-12520 (2000).

33. Chen, $P$. et al. The protective role of Per2 against carbon tetrachloride-induced hepatotoxicity. Am. J. Pathol. 174, 63-70 (2009).

34. Chen, J. et al. A comprehensive review of cytochrome P450 2E1 for xenobiotic metabolism. Drug Metab. Rev. 51, 178-195 (2019).

35. Rechnagel, R. O., Glende, E. A. \& Plaa, G. L. Carbon tetrachloride hepatotoxicity: an example of lethal cleavage. Crit. Rev. Toxicol. 2, 263-297 (1973).

36. Wong, W. Y., Chan, W. Y. \& Lee, S. T. Resistance to carbon tetrachlorideinduced hepatotoxicity in mice which lack CYP2E1 expression. Toxicol. Appl. Pharmacol. 153, 109-118 (1998).

37. Matsunaga, N. et al. Influence of feeding schedule on 24-h rhythm of hepatotoxicity induced by acetaminophen in mice. J. Pharmacol. Exp. Ther. 311, 594-600 (2004).

38. Cheung, C., Akiyama, T. E., Kudo, G. \& Gonzalez, F. J. Hepatic expression of cytochrome P450s in hepatocyte nuclear factor 1-alpha (HNF1a)-deficient mice. Biochem. Pharmacol. 66, 2011-2020 (2003).

39. Ktistaki, E. \& Talianidis, I. Modulation of hepatic gene expression by hepatocyte nuclear factor 1. Science 277, 109-112 (1997).

40. Wang, $T$. et al. Deletion of circadian gene Per1 alleviates acute ethanolinduced hepatotoxicity in mice. Toxicology 314, 193-201 (2013).

41. Kucera, N. et al. Unwinding the differences of the mammalian PERIOD clock proteins from crystal structure to cellular function. Proc. Natl. Acad. Sci. USA 109, 3311-3316 (2012).

42. Montagnese, S. et al. Sleep-wake abnormalities in patients with cirrhosis. Hepatology 59, 705-712 (2014) 